\title{
High-level expression of podoplanin in benign and malignant soft tissue tumors: Immunohistochemical and quantitative real-time RT-PCR analysis
}

\author{
YONGJUN XU ${ }^{1}$, AKIRA OGOSE ${ }^{1}$, HIROYUKI KAWASHIMA ${ }^{1}$, TETSUO HOTTA ${ }^{1}$, \\ TAKASHI ARIIZUMI ${ }^{1}$, GUIDONG LI $^{1}$, HAJIME UMEZU ${ }^{2}$ and NAOTO ENDO ${ }^{1}$ \\ ${ }^{1}$ Division of Orthopedic Surgery, Niigata University Graduate School of Medical and Dental Sciences, \\ 757-1, Asahimachi-dori, Niigata City, Niigata Prefecture 951-8510; ${ }^{2}$ Division of Pathology, Niigata University \\ Medical and Dental Hospital, 1-754, Asahimachi-dori, Chuo-ku, Niigata City, Niigata Prefecture 951-8520, Japan
}

Received August 31, 2010; Accepted November 30, 2010

DOI: $10.3892 /$ or.2011.1141

\begin{abstract}
Podoplanin is a $38 \mathrm{kDa}$ mucin-type transmembrane glycoprotein that was first identified in rat glomerular epithelial cells (podocytes). It is expressed in normal lymphatic endothelium, but is absent from vascular endothelial cells. D2-40 is a commercially available mouse monoclonal antibody which binds to an epitope on human podoplanin. D2-40 immunoreactivity is therefore highly sensitive and specific for lymphatic endothelium. Recent investigations have shown widespread applications of immunohistochemical staining with D2-40 in evaluating podoplanin expression as an immunohistochemical marker for diagnosis and prognosis in various tumors. To determine whether the podoplanin (D2-40) antibody may be useful for the diagnosis of soft tissue tumors, 125 cases, including 4 kinds of benign tumors, 15 kinds of malignant tumors and 3 kinds of tumor-like lesions were immunostained using the D2-40 antibody. Total RNA was extracted from frozen tumor tissue obtained from 41 corresponding soft tissue tumor patients and 12 kinds of soft tissue tumor cell lines. Quantitative real-time PCR reactions were performed. Immunohistochemical and quantitative real-time RT-PCR analyses demonstrated the expression of the podoplanin protein and mRNA in the majority of benign and malignant soft tissue tumors and tumor-like lesions examined, with the exception of alveolar soft part sarcoma, embryonal and alveolar rhabdomyosarcoma, extraskeletal Ewing's sarcoma/peripheral primitive neuro-ectodermal tumor and lipoma, which were completely negative for podoplanin. Since it is widely and highly expressed in nearly
\end{abstract}

Correspondence to: Dr Akira Ogose, Division of Orthopedic Surgery, Niigata University Graduate School of Medical and Dental Sciences, 757-1, Asahimachi-dori, Niigata City, Niigata Prefecture 951-8510, Japan

E-mail: aogose@med.niigata-u.ac.jp

Key words: podoplanin, soft tissue tumors, immunohistochemistry, quantitative real-time RT-PCR all kinds of soft tissue tumors, especially in spindle cell sarcoma, myxoid type soft tissue tumors and soft tissue tumors of the nervous system, podoplanin is considered to have little value in the differential diagnosis of soft tissue tumors.

\section{Introduction}

Podoplanin, also called the E11 antigen, is a $38 \mathrm{kDa}$ mucintype transmembrane glycoprotein that was first reported in lymphatic endothelial cells, epithelial cells of the choroid plexus, alveolar type I cells, osteoblasts, and peritoneal mesothelial cells by Wetterwald et al in 1996 (1). This protein was subsequently identified as a $43 \mathrm{kDa}$ protein on the surface of rat glomerular epithelial cells (podocytes), and because it was found to be involved in the flattening of foot processes in puromycin-induced nephrosis, it was named podoplanin (2). Recent investigations have demonstrated that podoplanin is regulated by the lymphatic-specific homeobox gene Prox 1, a master gene that controls the development of lymphatic progenitors from embryonic veins (3).

Podoplanin is homologous to the $\mathrm{hT} 1 \alpha-2$ (also known as Aggrus) gene which encodes the type I alveolar cell-specific antigen and to the oncofetal antigen M2A, which is recognized by the commercially available D2-40 antibody. Podoplanin promotes platelet aggregation, and possesses a platelet aggregation-stimulating (PLAG) domain. Thr52 is important for the activity of this domain (10). Tumor-induced platelet aggregation occurred early in the development of metastatic lesions, so it may be involved in cancer cell migration, invasion, metastasis, and malignant progression (4-12). Human podoplanin is expressed by several normal tissue cells, including: mesothelial cells $(13,14)$, osteocytes and osteoblasts $(1,13,15,16)$, stromal reticular cells, follicular dendritic cells of lymphoid tissue, ependymal cells of the central nervous system (CNS), and ovarian and testicular cells of both germ cell and sex-cord stromal origin, basal epithelial keratinocytes of the skin, cervix, and esophagus, and myoepithelial cells of the breast $(1,5,6,14,17-22)$. Podoplanin is a useful marker of Kaposi's sarcoma (22-24), 
and other vascular lesions, such as epithelioid hemangioendotheliomas of the head, neck and liver $(25,26)$, and hemangioblastomas of the CNS (27). Membranous and cytoplasmic immunoreactivity for podoplanin was detected in $86-100 \%$ of epithelioid mesotheliomas, respectively, whereas podoplanin is invariably negative in lung adenocarcinomas (14).

Strong podoplanin immunoreactivity was detected in primary and metastatic seminoma and testicular germ-cell tumors of young adults derived from intratubular germ cell neoplasia and carcinoma in situ $(6,28,29)$. Podoplanin expression is strongly expressed in pilocytic astrocytomas, glioblastomas, and primary central nervous system germ cell tumors (30-32). It is also strongly expressed in epidermal squamous cell carcinoma (33), oral leukoplakia (34-36), and skin adnexal carcinomas (37), whereas podoplanin is negative in metastatic adenocarcinomas and histiocytic neoplasms (38). The expression of podoplanin in a wide range of benign and malignant soft tissue tumors is not previously well characterized. In this study, we analyzed the expression of podoplanin in bengin and malignant soft tissue tumors and tumor-like lesions at both protein and mRNA level by immunohistochemical and quantitative real-time RT-PCR analysis.

\section{Materials and methods}

Tissue samples. Tumor specimens were obtained during surgery from 20 patients with undifferentiated high grade pleomorphic sarcomas [malignant fibrous histiocytomas (MFH), 20 pleomorphic types], 10 patients with myxofibrosarcoma, 31 patients with liposarcomas ( 8 well differentiated, 19 myxoid, and 4 dedifferentiated type 4 cases), 4 patients with biphasic synovial sarcomas, 7 patients with rhabdomyosarcomas (3 pleomorphics, 1 embryonal, and 3 alveolar type cases), 9 patients with malignant peripheral nerve sheath tumors, 3 patients with leiomyosarcomas, 3 patients with fibrosarcomas ( 2 adult cases, 1 sclerosing epithelioid case), 1 patient with extraskeletal chondrosarcoma, 3 patients with alveolar soft part sarcomas, 4 patients with epithelioid sarcomas, 3 patients with extraskeletal Ewing's sarcoma/ peripheral primitive neuroectodermal tumors (PNET), 4 patients with dermatofibrosarcoma protuberans, 6 patients with lipomas, 4 patients with schwannomas, 3 patients with fibromas, 3 patients with myxomas, 1 patient with pigmented villonodular synovitis (PVNS), 3 patients with ganglion tumors, and 3 patients with chronic expanding hematomas. Informed consent had been obtained previously from patients or their guardians. The tumor specimens were routinely fixed in $10 \%$ buffered formalin for $18-20 \mathrm{~h}$ at room temperature and paraffin-embedded. Sections $(4 \mu \mathrm{m})$ were cut and attached to APS coated glass slides. Hematoxylin and eosin was used for routine staining. The histology of these tissue samples was confirmed by experienced pathologists.

Cell lines and culture conditions. A human fibrosarcoma cell line (HT1080; Health Science Research Resources Bank), two human malignant fibrous histiocytoma cell lines (NMFH-1 and NMFH-2; Niigata University), two human synovial sarcoma cell lines (HS-SY-II; Dr H. Sonobe, Department of Pathology, Kochi Medical School, Kochi, Japan, and SYO-1;
Dr T. Ozaki, Okayama University), a human alveolar soft part sarcoma cell line (ASPS-KY; Dr S. Yanoma, Kanagawa Cancer Center, Yokohama, Japan), a human myxoid type liposarcoma cell line (402-92; Dr P. Åman, Department of Clinical Genetics, University Hospital, Lund, Sweden), a human malignant peripheral nerve sheath tumor cell line (NMS2; Niigata University), 3 human epithelioid sarcoma cell lines (SFT8606; Dr H. Iwasaki, Fukuoka University School of Medicine, Fukuoka, Japan, NEPS; from a patient with epithelioid sarcoma, and FU-EPS-1; Dr H. Iwasaki), and a human Ewing's/primitive neuroectodermal tumor (PNET) cell line (SKNMC; AmericanType Culture Collection, Bethesda, MD, USA) were incubated at $37^{\circ} \mathrm{C}$ in an atmosphere containing $5 \% \mathrm{CO}_{2}$ in culture media supplemented with $10 \%$ FBS, 0.6\% Kanamycin Sulfate (Gibco, Invitrogen), and 1\% Antibiotic-Antimycotic (Gibco, Invitrogen).

The NMFH-1, NMFH-2, HS-SY-II, ASPS-KY, 402-92, SFT8606, NEPS, FU-EPS-1, and NMS2 cells were cultured in RPMI-1640 culture medium (Gibco, Invitrogen), and the HT1080 and SKNMC cells were cultured in MEM- $\alpha$ medium (Gibco, Invitrogen); SYO-1 cells was cultured in medium DMEM (Gibco, Invitrogen). The medium was replaced twice a week. Culture cells were harvested for evaluation by quantitative real-time RT-PCR.

Quantitative real-time PCR. Total RNA was extracted from the frozen tumor tissue that has been obtained from 41 various soft tissue tumor patients, consisting of 3 cases of pleomorphic malignant fibrous histiocytomas, 4 myxoid malignant fibrous histiocytomas, 5 myxoid liposarcomas, 4 well differentiated liposarcomas, 1 case of dedifferentiated liposarcoma, 3 cases of malignant peripheral nerve sheath tumors, 2 synovial sarcomas, 2 dermatofibrosarcomas, 2 alveolar soft part sarcomas, 1 case each of rhabdomyosarcoma, leiomyosarcoma, fibrosarcoma, extraskeletal chondrosarcoma, epithelioid sarcoma, extraskeletal Ewing's sarcoma, 3 schwannomas, 4 lipomas, 1 fibroma and 1 case of pigmented villonodual synovitis, as well as soft tissue tumor-derived cell lines using Isogen (Nippongene, Toyama, Japan).

RNA was purified by standard techniques. The initial cDNA strand was synthesized using the PrimeScript ${ }^{\circledR}$ RT reagent kit (Takara, Shiga, Japan) according to the manufacturer's protocol. In brief, $0.5 \mu \mathrm{g}$ of total RNA was reversetranscribed at $37^{\circ} \mathrm{C}$ for $15 \mathrm{~min}$ in $10 \mu \mathrm{l}$ of $1 \mathrm{X}$ Prime Script buffer containing PrimeScript RT enzyme mix I, Random 6 mers $(100 \mu \mathrm{M})$ and Oligo dTPrimers $(50 \mu \mathrm{M})$, and RT enzyme was deactivated at $85^{\circ} \mathrm{C}$ for $5 \mathrm{sec}$. We performed PCR using the primers for podoplanin sequence. Total RNA from a normal human adult kidney (BioChain Institute, Hayward, USA) was used for calibration of the soft tissue tumors and their cell lines. Real-time PCR was performed using the Thermal Cycler Dice ${ }^{\circledR}$ Real Time system (Takara) with SYBR ${ }^{\circledR}$ Premix Ex Taq $\mu$ MTM II (Takara) following the manufacturer's instructions. Briefly, real-time PCR reactions were performed using the following program: $10 \mathrm{sec}$ at $94^{\circ} \mathrm{C}, 40$ cycles of $5 \mathrm{sec}$ at $95^{\circ} \mathrm{C}$ and $30 \mathrm{sec}$ at $60^{\circ} \mathrm{C}$, and finally $15 \mathrm{sec}$ at $95^{\circ} \mathrm{C}, 30 \mathrm{sec}$ at $60^{\circ} \mathrm{C}$ and $15 \mathrm{sec}$ at $95^{\circ} \mathrm{C}$ for dissociation. The results for each sample were normalized to glyceraldehyde-3-phosphate dehydrogenase (GAPDH). The data were analyzed using the software program provided by Takara. The results were 


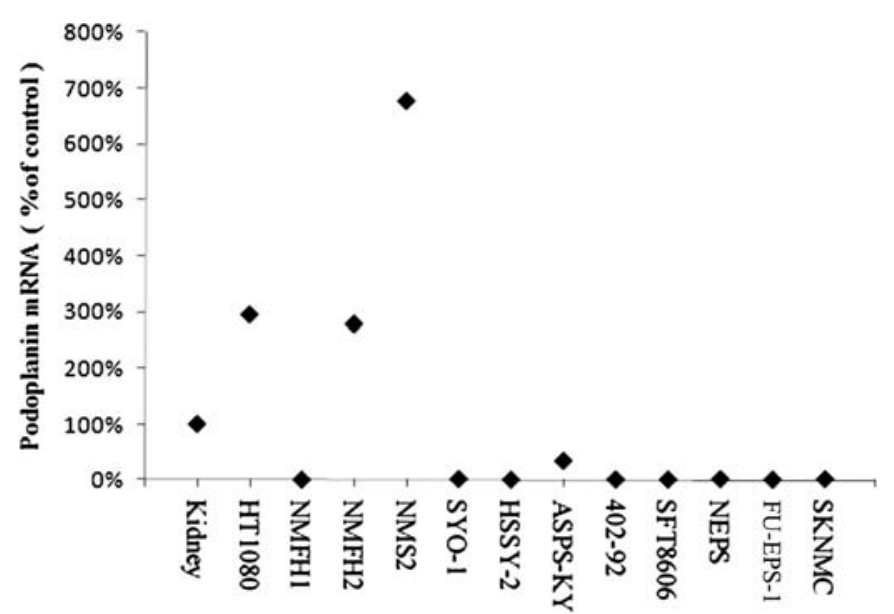

Figure 1. The expression of podoplanin mRNA in 12 kinds of soft tissue tumor cell lines, including a fibrosarcoma cell line (HT1080), 2 kinds of malignant fibrous histiocytomas (MFH) cell lines (NMFH1 and NMFH2), a malignant peripheral nerve sheath tumor cell line (NMS2), 2 kinds of synovial sarcoma cell lines (HS-SY-II and SYO-1), a alveolar soft part sarcoma cell line (ASPS-KY), a myxoid liposarcoma cell line (402-92), 3 kinds of epithelioid sarcoma cell lines (SFT8606, NEPS and FU-EPS-1), a Ewing's sarcoma/primitive neuroectodermal tumor (PNET) cell line (SKNMC). The normal human kidney was used for calibration. The results for each sample were normalized by the expression of glyceraldehydes-3phosphate dehydrogenase (GAPDH) as an internal standard. Podoplanin mRNA was expressed at higher levels in HT1080, NMFH2 and NMS2 than in control kidney specimen, whereas podoplanin was expressed at lower levels in other kinds of cell lines than in kidney.

expressed as normalized ratios. The Primers for podoplanin and GAPDH (Table III) were designed and validated using a Perfect Real-time Primer Support System (Takara).

Immunohistochemical analysis. Immunohistochemical analysis of podoplanin in soft tissue tumors was performed. Tissue sections $(4 \mu \mathrm{m})$ from paraffin-embedded soft tissue tumor specimens were mounted on APS coated glass slides. In brief, the tissue sections were deparaffinized in xylene and rehydrated in graded alcohols. Slides were steamed for antigen retrieval with $10 \mathrm{mM}$ citrate buffer at $\mathrm{pH} 6.0$ (Dako Cytomation, Carpinteria, CA) at $85-100^{\circ} \mathrm{C}$ for $40 \mathrm{~min}$. Part of the samples were treated with $0.2 \%$ trypsin solution at $37^{\circ} \mathrm{C}$ for $20 \mathrm{~min}$ for antigen retrieval to avoid damage and exfoliation of the samples by the hot steam.

After cooling for $15 \mathrm{~min}$, the slides were immersed in methanol containing $0.3 \%$ hydrogen peroxide for $20 \mathrm{~min}$ at room temperature to block the endogenous peroxidase activity, and then were incubated with phosphate buffered saline containing 10\% goat serum (Dako, Denmark) for $20 \mathrm{~min}$ at room temperature to reduce nonspecific reactions. The slides were then incubated with the monoclonal mouse antibody (D2-40, Dako) against human podoplanin at a 1:100 dilution at $4^{\circ} \mathrm{C}$ overnight, followed by reacting them with a universal immunoperoxidase polymer, anti-mouse and anti-rabbit (Histofine Simple Stain MAX PO (MULTI); Nichirei, Tokyo, Japan) antibodies for $30 \mathrm{~min}$ at room temperature. The peroxidase activity was detected with 3'-diaminobenzidine tetrahydrochloride (Nichirei). The slides were counterstained with hematoxylin (Vector Laboratories, Inc. Burlingame, CA).
For histological scoring, representative areas of each tissue section were selected and evaluated independently by 2 investigators, who were blinded to the clinical information pertaining to the subjects. The immunostaining was graded on a sliding scale of 0 to $3+$ according to the perentage of reactive cells as follows: 0 , no staining, $1+, 0-10 \%$ cells stained with weak intensity, $2+; 10-40 \%$ cells stained with variable intensity, $3+;>40 \%$ of cells stained with strong intensity.

\section{Results}

The expression of podoplanin mRNA in soft tissue tumor cell lines. To quantify the expression of podoplanin mRNA in human soft tissue tumor cell lines, we performed quantitative real-time PCR analyses of 12 soft tissue tumor cell lines. The relative podoplanin mRNA expression levels of each tumor are shown in Fig. 1. Podoplanin transcript levels in the HT1080, NMFH-2 and NMS2 tumor cell lines were significantly higher than in the control kidney specimen. In contrast, the podoplanin transcript levels in the other tumor cell lines were lower than in the kidney.

The expression of podoplanin mRNA in soft tissue tumors. To quantify the expression of podoplanin mRNA in human soft tissue tumors, we performed quantitative real-time PCR analyses of soft tissue tumors from 42 patients. The relative podoplanin mRNA expression levels of each tumor are shown in Figs. 2 and 3. Podoplanin transcript levels in a wide range of benign and malignant soft tissue tumors, as well as tumorlike lesions were significantly higher than in the control kidney specimen. Only in primitive neuroectodermal tumor, alveolar soft part sarcomas, lipomas, some liposarcomas (well differentiated type), was podoplanin mRNA expressed at a lower level.

Immunohistochemical analysis of podoplanin in malignant soft tissue tumors. High-level podoplanin expression was observed in various types of malignant soft tissue tumors when the anti-podoplanin antibody (D2-40) was used, especially in spindle cell sarcoma, myxoid type and the nervous system soft tissue tumors, whereas round cell sarcoma exhibited no reactivity for D2-40 and was completely negative for podoplanin. The immunohistochemical results are summarized in Table I and Fig. 4. Podoplanin immunoreactivity was detected in 17 of 20 (85\%) tumors. In four of the cases of undifferentiated high grade pleomorphic sarcomas (pleomorphic malignant fibrous histiocytomas); the staining was graded as $3+, 2+$ in six cases, and as $1+$ in the other seven of these.

Pleomorphic malignant fibrous histiocytomas showed membranous immunoreactivity for D2-40. In 3 cases, there was no detctedable podoplanin expression. Immunoreactivity to D2-40 was observed in all of the samples of myxofibrosarcoma $(10 / 10,100 \%)$. In 5 of these cases, the staining was graded as $3+$, in 2 as $2+$, and in 3 as $1+$. The reaction with the D2-40 antibody was characterized by a strong, thick membranous staining pattern. Among liposarcomas, the well differentiated types showed weak immunoreactivity to D240 , and only 1 of $8(12.5 \%)$ was considered positive. The 


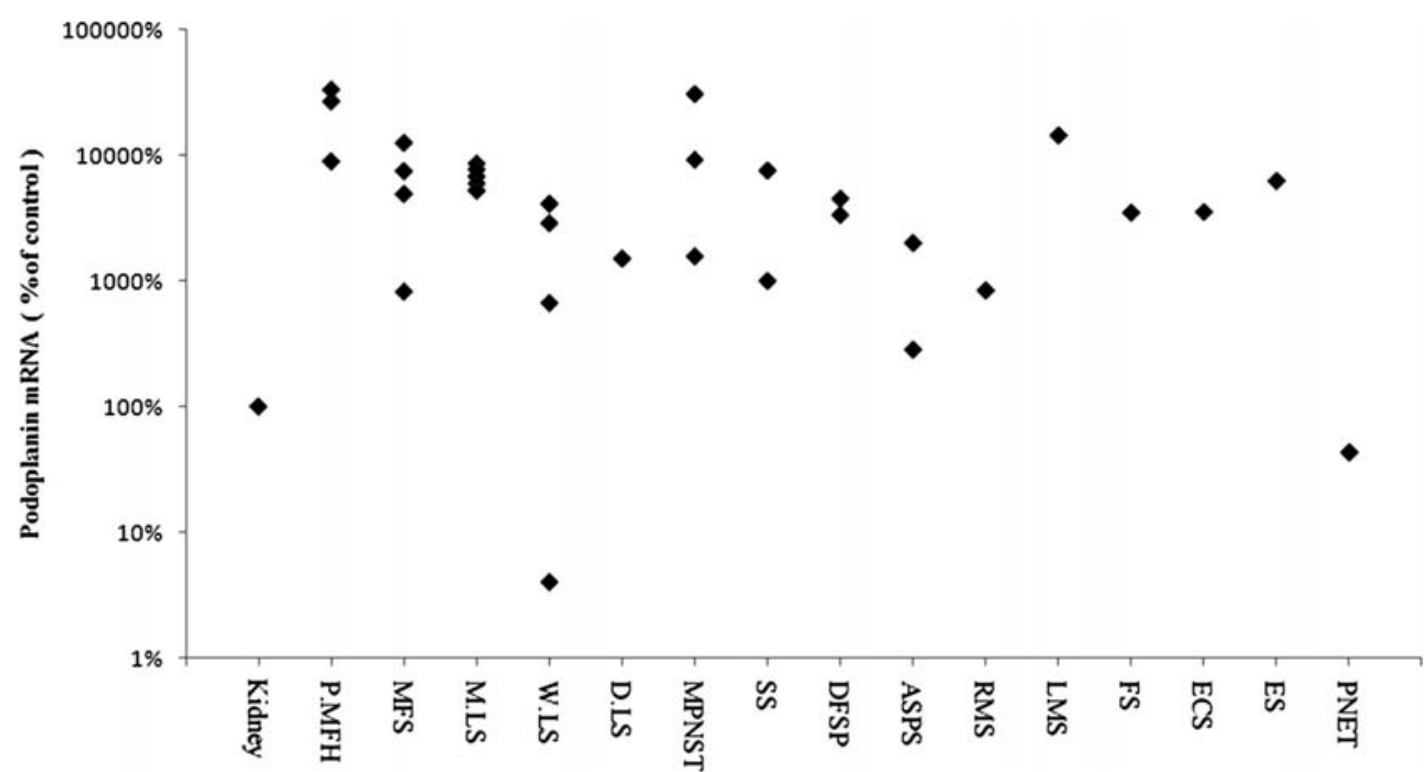

Figure 2. The expression of podoplanin mRNA in 15 kinds of malignant soft tissue tumors. The normal human kidney was used for calibration. The results for each sample were normalized by the expression of glyceraldehydes-3-phosphate dehydrogenase (GAPDH) as an internal standard. The expression of podoplanin mRNA in 3 undifferentiated high grade pleomorphic sarcomas (pleomorphic malignant fibrous histiocytomas) and 4 myxofibrosarcomas. Podoplanin mRNA was expressed at higher levels in all 3 pleomorphic MFH and 4 myxofibrosarcomas than in kidney. P.MFH, pleomorphic malignant fibrous histiocytoma; MFS, myxofibrosarcoma. The expression of podoplanin mRNA in 5 myxoid liposarcomas, 4 well differentiated liposarcomas and in 1 case of dedifferentiated liposarcoma. Podoplanin mRNA was expressed at higher levels in almost all the cases than in kidney except for W.LS4, which expressed podoplanin at lower level than in kidney. M.LS, myxoid liposarcoma; W.LS, well differentiated liposarcoma; D.LS, dedifferentiated liposarcoma. The expression of podoplanin mRNA in 3 malignant peripheral nerve sheath tumors, 2 biphasic synovial sarcomas, 2 dermatofibrosarcoma protuberans and 2 cases alveolar soft part sarcomas. Podoplanin mRNA was expressed at higher levels in all the cases than in kidney. MPNST, malignant peripheral nerve sheath tumors; SS, biphasic synovial sarcoma; DFSP, dermatofibrosarcoma protuberans; ASPS, alveolar soft part sarcoma. The expression of podoplanin mRNA in 1 rhabdomyosarcoma, 1 leiomyosarcoma, 1 fibrosarcoma, 1 extraskeletal chondrosarcoma, 1 epithelioid sarcoma and 1 case of extraskeletal Ewing's sarcoma/peripheral primitive neuroectodermal tumor. Podoplanin mRNA was expressed at higher levels in almost all the cases than in kidney except for PNET, which expressed podoplanin at lower level than in kidney. RMS, Rhabdomyosarcoma; LMS, leiomyosarcoma; FS, fibrosarcoma; ECS, extraskeletal chondrosarcoma; ES, epithelioid sarcoma; PNET, extraskeletal Ewing's sarcoma/peripheral primitive neuroectodermal tumor.

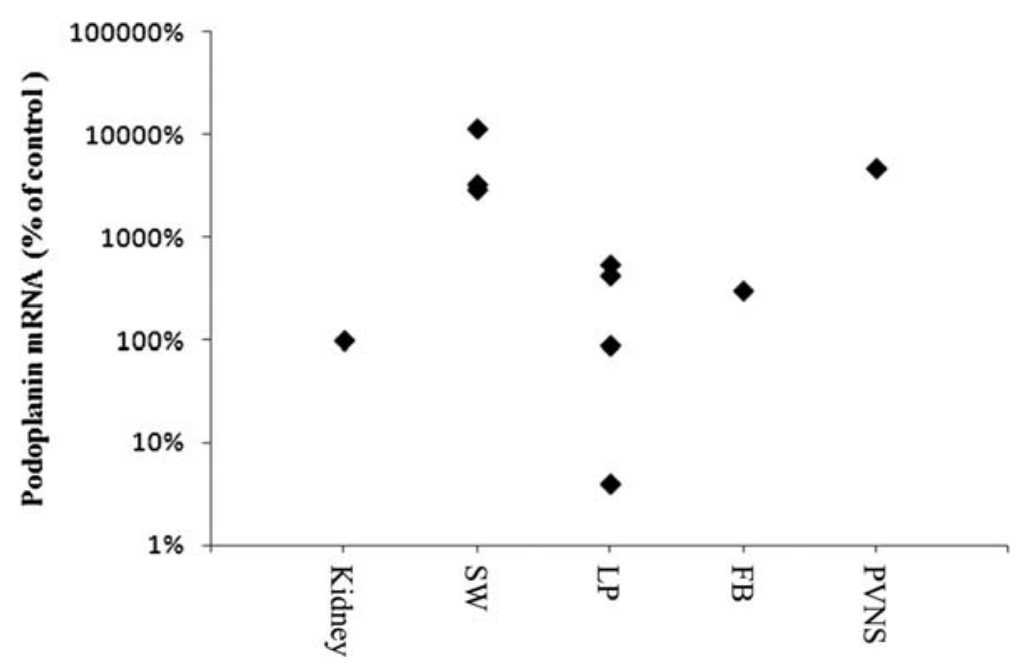

Figure 3. The expression of podoplanin mRNA in 3 types of benign soft tissue tumors and a tumor-like lesion. The normal human kidney was used for calibration. The results for each sample were normalized by the expression of glyceraldehydes-3-phosphate dehydrogenase (GAPDH) as an internal standard. Podoplanin mRNA was expressed at higher levels in 3 schwannomas, 2 lipomas, 1 fibroma and 1 pigmented villonodular synovitis than in kidney, whereas podoplanin was expressed at lower levels in 2 lipomas (LP1 and LP4) than in kidney. SW, schwannoma; LP, lipoma; FB, fibroma; PVNS, pigmented villonodular synovitis.

staining was graded as $1+$ and the reaction was characterized by a dot-like membranous staining pattern.

In contrast, myxoid types showed strong immunoreactivity to D2-40, and 18 of $19(94.7 \%)$ tumors exhibited positive reactivity. The staining was graded in 11 cases as $3+$, in 5 as $2+$, in 2 as $1+$. The reaction with the D2-40 antibody was characterized by a strong, thick membranous staining pattern. Podoplanin immunoreactivity was detected 
Table I. D2-40 (podoplanin) immunoreactivity in malignant soft tissue tumors.

\begin{tabular}{|c|c|c|c|c|c|c|c|}
\hline \multirow[b]{2}{*}{ Tumor type } & \multirow[b]{2}{*}{$\begin{array}{l}\text { No. } \\
\text { of cases }\end{array}$} & \multicolumn{4}{|c|}{ Grade of reactivity ${ }^{\mathrm{a}}$} & \multirow[b]{2}{*}{$\begin{array}{c}\text { No. }(\%) \text { of } \\
\text { positive cases }\end{array}$} & \multirow[b]{2}{*}{$\begin{array}{l}\text { Average } \\
\text { grade }\end{array}$} \\
\hline & & 0 & $1+$ & $2+$ & $3+$ & & \\
\hline \multicolumn{8}{|l|}{ Malignant fibrous histiocytoma } \\
\hline $\begin{array}{l}\text { Undifferentiated high grade } \\
\text { pleomorphic sarcoma (Pleomorphic MFH) }\end{array}$ & 20 & 3 & 7 & 6 & 4 & $17(85)$ & 1.55 \\
\hline Myxofibrosarcoma & 10 & 0 & 3 & 2 & 5 & $10(100)$ & 2.20 \\
\hline \multicolumn{8}{|l|}{ Liposarcoma } \\
\hline Well differentiated & 8 & 7 & 1 & 0 & 0 & $1(12.5)$ & 0.125 \\
\hline Myxoid & 19 & 1 & 2 & 5 & 11 & $18(94.7)$ & 2.37 \\
\hline Dedifferentiated & 4 & 0 & 1 & 3 & 0 & $4(100)$ & 1.75 \\
\hline \multicolumn{8}{|l|}{ Synovial sarcoma } \\
\hline Biphasic & 4 & 0 & 1 & 0 & 3 & $4(100)$ & 2.50 \\
\hline \multicolumn{8}{|l|}{ Rhabdomyosarcoma } \\
\hline Pleomorphic & 3 & 0 & 1 & 2 & 0 & $3(100)$ & 1.67 \\
\hline Embryonal & 1 & 1 & 0 & 0 & 0 & $0(0)$ & 0.00 \\
\hline Alveolar & 3 & 3 & 0 & 0 & 0 & $0(0)$ & 0.00 \\
\hline Malignant peripheral nerve sheath tumor & 9 & 0 & 4 & 4 & 1 & $9(100)$ & 1.67 \\
\hline Leiomyosarcoma & 3 & 0 & 1 & 0 & 2 & $3(100)$ & 2.33 \\
\hline Fibrosarcoma & 3 & 0 & 1 & 0 & 2 & $3(100)$ & 2.33 \\
\hline Extraskeletal chondrosarcoma & 1 & 0 & 0 & 1 & 0 & $1(100)$ & 2.00 \\
\hline Alveolar soft part sarcoma & 3 & 3 & 0 & 0 & 0 & $0(0)$ & 0.00 \\
\hline Epithelioid sarcoma & 4 & 3 & 1 & 0 & 0 & $1(25)$ & 0.25 \\
\hline $\begin{array}{l}\text { Extraskeletal Ewing's sarcoma/ } \\
\text { peripheral primitive neuroectodermal tumor }\end{array}$ & 4 & 2 & 0 & 1 & 1 & $0(0)$ & 0.00 \\
\hline Dermatofibrosarcoma protuberans & 4 & 2 & 0 & 1 & 1 & $2(50)$ & 1.25 \\
\hline
\end{tabular}

${ }^{\mathrm{a}} 0$, no staining; $1+, 0-10 \%$ tumor cell reactive; $2+, 10-40 \%$ tumor cell reactive; $3+,>40 \%$ tumor cell reactive.

in 4 of $4(100 \%)$ of the dedifferentiated type tumors. In 3 of these cases, the staining was graded as $2+$, and in 1 as $1+$. The reaction was characterized by a strong, thick staining pattern in dedifferentiated areas. Podoplanin immunoreactivity was detected in 4 of $4(100 \%)$ biphasic synovial sarcomas; the staining was graded in 3 cases as $3+$, and in 1 as $1+$. The reaction showed a dot-like, diffuse membranous staining pattern. Three of three pleomorphic rhabdomyosarcomas (100\%) showed positive reactivity for the D2-40 antibody. The staining was graded $2+$ in 2 cases, and in 1 case as $1+$. The reaction was characterized by a diffuse, dot-like membranous staining pattern, whereas three of the alveolar and one embryonal rhabdomyosarcoma were completely negative for D2-40.

Podoplanin immunoreactivity was detected in 9 of 9 $(100 \%)$ malignant peripheral nerve sheath tumors. The staining was graded in 1 case as $3+$, in 4 as $2+$, and in 4 as $1+$. The reaction showed a diffuse, dot-like membranous staining pattern. Three of three leiomyosarcomas (100\%) showed positive reactivity for D2-40. The staining was graded in 2 cases as $3+$, and in 1 as $1+$. The reaction was characterized by a strong, diffuse, ring-like staining pattern. Among the fibrosarcomas, 2 of 2 adult types and 1 of 1 sclerosing epithelioid tumor showed positive reactivity for D2-40. The staining of adult types was graded in 1 case as $3+$, and in 1 as $1+$, and the sclerosing epithelioid type was graded in 1 case as $3+$. The reaction of sclerosing epithelioid type was characterized by a strong, diffuse cytoplasmic staining pattern. Podoplanin immunoreactivity was detected in 1 case of extraskeletal chondrosarcoma. The staining was graded as $2+$ and showed a discontinuous membranous staining pattern.

One of four epithelioid sarcomas (25\%) exhibited positive reactivity for D2-40. The staining was graded in one cases as $1+$, and as 0 in the other three of the tumors. The reaction was characterized by a dot- and ring-like staining pattern for D2-40. Two of the four dermatofibrosarcoma protuberans $(50 \%)$ showed positive reactivity for D2-40, and the staining was graded in 1 case as $3+$, and in the other case as $2+$. The reaction was characterized by a strong, diffuse and ring-like pattern for D2-40. None of the alveolar soft part sarcomas, 


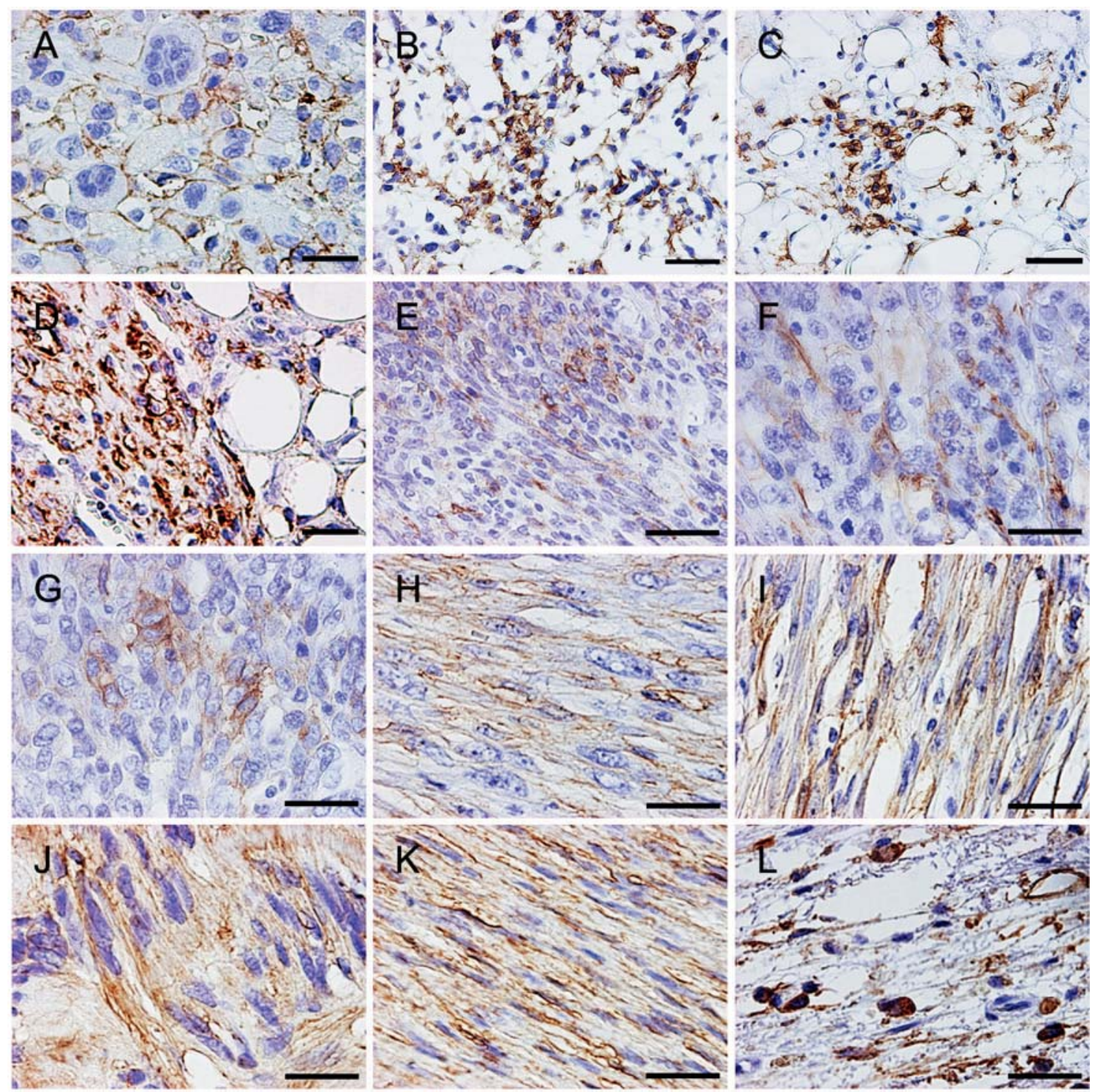

Figure 4. Strong podoplanin immunoreactivity in malignant and benign soft tissue tumors. Podoplanin expression was determined by the anti-podoplanin antibody D2-40. (A) Pleomorphic malignant fibrous histiocytomas showed membranous immunoreactivity for D2-40. (B) Myxofibrosarcomas showed strong, thick membranous staining pattern. (C) Myxoid liposarcomas showed strong, thick membranous staining pattern. (D) Dedifferentiated liposarcoma showed strong, thick staining pattern, especially in dedifferentiated areas. (E) Biphasic synovial sarcomas showed dot-like, diffuse membranous staining pattern. (F) Pleomorphic rhabdomyosarcomas showed diffuse, dot-like membranous staining pattern. (G) Malignant peripheral nerve sheath tumors showed diffuse, dotlike membranous staining pattern. (H) Leiomyosarcomas showed strong, diffuse, ring-like staining pattern. (I) Fibrosarcoma showed a strong, diffuse cytoplasmic staining pattern. (J) Schwannoma were a strong, diffuse, ring-like cytoplasmic staining pattern. (K) Fibroma showed a strong, diffuse, ring-like staining pattern. (L) Myxoma showed a strong, diffuse, ring-like cytoplasmic staining pattern for D2-40. Bar, (A-D) $100 \mu \mathrm{m}$; (E-L) $50 \mu \mathrm{m}$.

embryonal and alveolar rhabdomyosarcomas, nor the extraskeletal Ewing's sarcoma/peripheral primitive neuroectodermal tumors (PNET) exhibited reactivity for D2-40, and all were completely negative for podoplanin.

Immunohistochemical analysis of podoplanin in benign soft tissue tumors and tumor-like lesions. The immunohistochemical results are summarized in Table II and Fig. 5. Two of three cases of myxoma showed positive reactivity for D2-40, and staining was graded in these 2 cases as $3+$. The reaction showed a strong, diffuse, ring-like cytoplasmic staining pattern for D2-40. Four of four (100\%) schwannomas exhibited positive reactivity for D2-40. The staining was graded in 3 cases as $3+$, and in 1 case as $2+$. The reaction was characterized by a strong, diffuse, ring-like cytoplasmic staining pattern. Two of three cases $(66.7 \%)$ of fibromas showed positive reactivity for D2-40. The staining was graded in 1 case as $3+$, and in 1 case as $1+$, and showed a strong, diffuse, ring-like staining pattern. The lipoma exhibited no reactivity for D2-40, indicating that it was completely negative for podoplanin. Among the tumor-like lesions, 1 case of pigmented villonodular synovitis (PVNS) showed positive reactivity, and the staining was graded as $3+$. The reaction exhibited discontinuous, and a diffuse membranous staining pattern was observed. The ganglion tumors and chronic expand expanding hematoma exhibited positive reactivity for D2-40. The staining of the ganglion tumors was graded as $3+$ in one case, and as $2+$ in the other two, and similar staining patterns were present in chronic expand expanding hematoma. 
Table II. D2-40 (podoplanin) immunoreactivity in benign soft tissue tumors and tumor-like lesions.

\begin{tabular}{|c|c|c|c|c|c|c|c|}
\hline \multirow[b]{2}{*}{ Tumor and tumor-like lesion type } & \multirow[b]{2}{*}{$\begin{array}{c}\text { No. } \\
\text { of cases }\end{array}$} & \multicolumn{4}{|c|}{ Grade of reactivity $^{\mathrm{a}}$} & \multirow[b]{2}{*}{$\begin{array}{c}\text { No. }(\%) \text { of } \\
\text { positive cases }\end{array}$} & \multirow[b]{2}{*}{$\begin{array}{c}\text { Average } \\
\text { grade }\end{array}$} \\
\hline & & 0 & $1+$ & $2+$ & $3+$ & & \\
\hline Lipoma & 6 & 6 & 0 & 0 & 0 & $0(0)$ & 0.00 \\
\hline Schwannoma & 4 & 0 & 0 & 1 & 3 & $4(100)$ & 2.75 \\
\hline Fibroma & 3 & 1 & 1 & 0 & 1 & $2(66.7)$ & 1.33 \\
\hline Myxoma & 3 & 1 & 0 & 0 & 2 & $2(66.7)$ & 2.00 \\
\hline Pigmented villonodular synovitis & 1 & 0 & 0 & 0 & 1 & $1(100)$ & 3.00 \\
\hline Ganglion & 3 & 0 & 0 & 2 & 1 & $3(100)$ & 2.33 \\
\hline Chronic expanding hematoma & 3 & 0 & 0 & 2 & 1 & $3(100)$ & 2.33 \\
\hline
\end{tabular}

${ }^{\mathrm{a}} 0$, no staining; $1+, 0-10 \%$ tumor cell reactive; $2+, 10-40 \%$ tumor cell reactive; $3+,>40 \%$ tumor cell reactive.
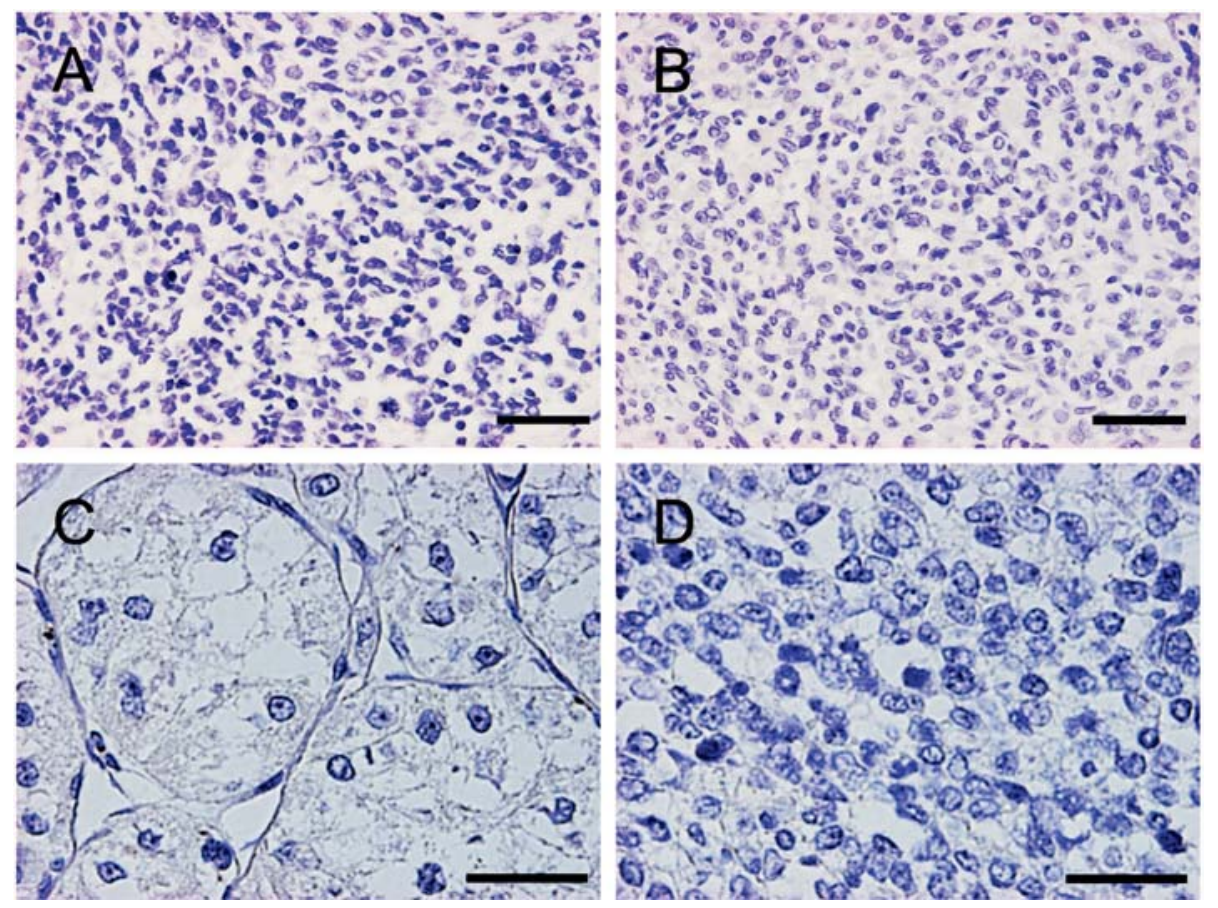

Figure 5. No podoplanin immunoreactivity in soft tissue sarcomas. Podoplanin expression was determined by the anti-podoplanin antibody D2-40. (A) Alveolar rhabdomyosarcomas, (B) embryonal rhabdomyosarcoma, (C) alveolar soft part sarcomas, (D) extraskeletal Ewing's sarcoma/peripheral primitive neuroectodermal tumor (PNET). None of (A-C), nor (D) exhibited reactivity for D2-40, and all were completely negative for podoplanin. Bar, (A and B) $100 \mu \mathrm{m} ;(\mathrm{C}$ and D) $50 \mu \mathrm{m}$.

\section{Discussion}

Jokinen et al (39) reported that 16 cases of 21 (76\%) schwannomas showed strong and diffuse cytoplasmic positive reactivity for D2-40, and 3 of 4 cases (75\%) of epithelioid malignant peripheral nerve sheath tumors showed a strong, diffuse membranous staining pattern for D2-40, which were similar to our immunohistochemical staining results in the same tumor types. Ordóñez et al (14) reported that 4 of 6 biphasic synovial sarcoma $(66.7 \%)$ showed positive reactivity for podoplanin in the epithelioid component of the tumor, which was also similar to our immunohistochemical staining results in the same type of tumors. Although podoplanin expression has been detected in various human tumors, the expression of podoplanin in the majority of benign and malignant soft tissue tumors has not been well examined, except for schwannoma, epithelioid malignant peripheral nerve sheath tumors, and biphasic synovial sarcoma.

In the present study, immunohistochemical and quantitative real-time RT-PCR analyse demonstrated that there is expression of podoplanin at the protein and mRNA levels in the majority of benign and malignant soft tissue tumors and tumor-like lesions. Furthermore, it seemed that podoplanin immunoreactivity showed a very high correlation with cell morphology in soft tissue sarcoma. Spindle cell soft tissue sarcoma exhibited relatively strong immunoreactivity for D2-40 and was positive for podoplanin. We found that in 10 $(100 \%)$ of 10 myxofibrosarcomas, in $18(94.7 \%)$ of 19 
Table III. Primers used for quantitative real-time polymerase chain reaction.

\begin{tabular}{lllc}
\hline Name & \multicolumn{1}{c}{ Sequence } & Primer ID & GenBank accession no. \\
\hline Podoplanin & 5'-TGACTCCAGGAACCAGCGAAG-3' & HA071355-F & NM_006474 \\
& 5'-GCGAATGCCTGTTACACTGTTGA-3' & HA071335-R & \\
GAPDH & 5'-GCACCGTCAAGGCTGAGAAC-3' & HA067812-F & NM_002046 \\
& 5'-TGGTGAAGACGCCAGTGGA-3' & HA067812-R & \\
\hline
\end{tabular}

GAPDH, glyceraldehydes-3-phosphate dehydrogenase.

myxoid liposarcomas, in $4(100 \%)$ of 4 dedifferentiated liposarcomas, in $4(100 \%)$ of 4 biphasic synovial sarcomas, in $3(100 \%)$ of 3 leiomyosarcomas, in $3(100 \%)$ of 3 pleomorphic rhabdomyosarcomas, in $9(100 \%)$ of 9 malignant peripheral nerve sheath tumors, and in $3(100 \%)$ of 3 fibrosarcomas, there was widespread immunoreactivity for podoplanin with variable levels of intensity, frequency and staining patterns in most of the malignant soft tissue tumor types. In contrast, there was only limited and weak staining in $1(12.5 \%)$ of 8 well differentiated liposarcomas and in $2(50 \%)$ of 4 dermatofibrosarcoma protuberans. No immunoreactivity was seen in any of the 3 alveolar soft part sarcomas, the 3 extraskeletal Ewing's sarcoma/peripheral primitive neuroectodermal tumors, the 1 embryonal rhabdomyosarcoma or any of the 3 alveolar rhabdomyosarcomas, which demonstrated that round cell soft tissue sarcomas exhibited no immunoreactivity for podoplanin. Similar staining patterns were seen in benign soft tissue tumors and tumor-like lesions.

There was positive reactivity to the D2-40 antibody in 2 $(66.7 \%)$ of 3 myxomas, in $4(100 \%)$ of 4 schwannoma, in 2 $(66.7 \%)$ of 3 fibroma. Strong, diffuse expression of podoplanin protein has been also found in all tumor-like lesions, including the 1 pigmented villonodular synovitis, 3 ganglion tumors and the 3 chronic expanding hematomas. It has been reported that podoplanin induces an actin cytoskeleton rearrangement by increasing the epithelial-mesenchymal transition (EMT) based on the binding activity with plasma membrane protein ERM (ezrin, radixin and moesin) to activate RhoA. This series of events in podoplanin-positive cancer cells may facilitate EMT, which plays a role in the plasma membrane extension and actin cytoskeleton rearrangement (40-42).

In this study, quantitative real-time RT-PCR analyses confirmed that almost all of the tumor tissue that was examined contained podoplanin mRNA transcripts at a level higher than that in human kidney, except for 1 case of well differentiated liposarcoma, 1 case of extraskeletal Ewing's sarcoma/peripheral primitive neuroectodermal tumor, and 2 lipomas. The results are in accord with the ubiquitous expression of the podoplanin protein in the immunohistochemical staining results of the corresponding tumors. Our findings demonstrated that the majority of benign and malignant soft tissue tumors and tumor-like lesions express podoplanin mRNA at a relatively high level. Therefore, it seems that podoplanin expression cannot be used to distinguish benign and malignant soft tissue tumors.

Although most of the malignant soft tissue tumors were confirmed to have strong expression levels of both podoplanin
mRNA and protein, the majority of their corresponding soft tissue cell lines had lower expression of podoplanin mRNA. The expression of podoplanin in the HT1080 human fibrosarcoma cell line was reported at a low level unless the cells were stimulated with transforming growth factor- $\beta$, thus suggesting that podoplanin mRNA is translated to podoplanin protein by complex biological regulation $(43,44)$.

High-level expression of podoplanin at the mRNA transcriptional level was detected in almost all of the different types of soft tissue sarcoma cells. Podoplanin mRNA might thus be detected in rare viable soft tissue tumors in the peripheral blood circulation. The use of reverse transcriptase-polymerase chain reaction (RT-PCR) for determination of the podoplanin mRNA level, and the realtime quantitative amount of podoplanin mRNA in the peripheral blood may reflect the size and dissemination of the tumor, and the level of the relative quantitative podoplanin mRNA may be reflect responses to treatment and prognosis of tumors, such as the likelihood of late recurrence, longterm survival, and can represent a rapid and precise method for determination of the response to chemotherapy or radiotherapy. Thus, the podoplanin mRNA RT-PCR assay of viable soft tissue tumor cells in the peripheral blood of cancer patients might be of some value as for follow-up and treatment monitoring of soft tissue sarcomas.

In conclusion, podoplanin is widely expressed at a high level in various benign and malignant soft tissue tumors, especially in spindle cell sarcoma, myxoid type and soft tissue tumors of the nervous system, whereas round cell sarcoma exhibited no reactivity for the D2-40 antibody and were completely negative for podoplanin. We did not find any valuable tissue-specificity for the differential diagnosis of soft tissue tumors. Thus, our results suggest that podoplanin has little value in the diagnosis of soft tissue tumors. However, evaluation of the podoplanin mRNA of circulating soft tissue tumor cells might be of some value for follow-up and monitoring the treatment response of soft tissue sarcomas.

\section{References}

1. Wetterwald A, Hofstetter W, Cecchini MG, et al: Characterization and cloning of the E11 antigen, a marker expressed by rat osteoblasts and osteocytes. Bone 18: 125-132, 1996.

2. Breiteneder-Geleff S, Matsui K, Soleiman A, et al: Podoplanin, novel 43-kd membrane protein of glomerular epithelial cells, is down-regulated in puromycin nephrosis. Am J Pathol 151: 1141-1152. 1997. 
3. Hong YK, Harvey N, Noh YH, Schacht V, Hirakawa S and Detmar M: Prox1 is a master control gene in the program specifying lymphatic endothelial cell fate. Dev Dyn 225: 351-357, 2002.

4. Marks A, Sutherland DR, Bailey D, et al: Characterization and distribution of an oncofetal antigen (M2A antigen) Expressed on testicular germ cell tumours. Br J Cancer 80: 569-578, 1999.

5. Schacht V, Ramirez MI, Hong YK, et al: T1a/podoplanin deficiency disrupts normal lymphatic vasculature formation and causes lymphedema. EMBO J 22: 3546-3556, 2003.

6. Schacht V, Dadras SS, Johnson LA, Jackson DG, Hong YK and Detmar M: Up-regulation of the lymphatic marker podoplanin, a mucin-type transmembrane glycoprotein, in human squamous cell carcinomas and germ cell tumors. Am J Pathol 166: 913-921, 2005

7. Kato Y, Kaneko M, Sata M, Fujita N, Tsuruo T and Osawa M: Enhanced expression of Aggrus (T1alpha/podoplanin), a plateletaggregation-inducing factor in lung squamous cell carcinoma. Tumour Biol 26: 195-200, 2005.

8. Suzuki-Inoue $\mathrm{K}$, Kato $\mathrm{Y}$, Inoue $\mathrm{O}$, et al: Involvement of the snake toxin receptor CLEC-2, in podoplanin-mediated platelet activation, by cancer cells. J Biol Chem 282: 25993-26001, 2007

9. Kato Y, Kaneko MK, Kunita A, et al: Molecular analysis of the pathophysiological binding of the platelet aggregation-inducing factor podoplanin to the C-type lectin-like receptor CLEC-2. Cancer Sci 99: 54-61, 2008

10. Kaneko MK, Kato Y, Kameyama A, et al: Functional glycosylation of human podoplanin: Glycan structure of platelet aggregation-inducing factor. FEBS Lett 581: 331-336, 2007.

11. Kato Y, Fujita N, Kunita A, Sato S, Kaneko M and Osawa M: Molecular identification of Aggrus/T1a as a platelet aggregationinducing factor expressed in colorectal tumors. J Biol Chem 278: 51599-51605, 2003.

12. Kato Y, Kaneko MK, Kuno A, et al: Inhibition of tumor cellinduced platelet aggregation using a novel anti-podoplanin antibody reacting with its platelet-aggregation-stimulating domain. Biochem Biophys Res Commun 349: 1301-1307, 2006.

13. Ordóñez NG: Podoplanin: a novel diagnostic immunohistochemical marker. Adv Anat Pathol 3: 83-88, 2006.

14. Ordóñez NG: D2-40 and podoplanin are highly specific and sensitive immuno-histochemical markers of epithelioid malignant mesothelioma. Hum Pathol 36: 372-380, 2005.

15. Hantusch B, Kalt R, Krieger S, Puri C and Kerjaschki D: Sp1/Sp3 and DNAmethylation contribute to basal transcriptional activation of human podoplanin in MG63 versus Saos-2 osteoblastic cells. BMC Mol Biol 8: 20, 2007.

16. Zhang K, Barragan-Adjemian C, Ye L, et al: E11/gp38 selective expression in osteocytes: Regulation by mechanical strain and role in dendrite elongation. Mol Cell Biol 26: 4539-4552, 2006.

17. Williams MC, Cao Y, Hinds A, Rishi AK and Wetterwald A: T1alpha protein is developmentally regulated and expressed by alveolar type I cells, choroid plexus and ciliary epithelia of adult rats. Am J Respir Cell Mol Biol 14: 577-585, 1996.

18. Ramirez MI, Millien G, Hinds A, Cao YX, Seldin DC and Williams MC: T1alpha, a lung type I cell differentiation gene, is required for normal lung cell proliferation and alveolous formation at birth. Dev Biol 256: 61-72, 2003.

19. Yu H, Gibson JA, Pinkus GS and Hornick JL: Podoplanin (D240 ) is a novel marker for follicular dendritic cell tumors. Am J Clin Pathol 128: 776-782, 2007.

20. Raica M, Cimpean AM and Ribatti D: The role of podoplanin in tumor progression and metastasis. Anticancer Res 28: 2997-3006, 2008.

21. Marsee DK, Pinkus GS, Hornick JL: Podoplanin (D2-40) is a highly effective marker of follicular dendritic cells. Appl Immunohistochem Mol Morphol 17: 102-107, 2009.

22. Breiteneder-Geleff S, Soleiman A, Kowalski H, et al: Angiosarcomas express mixed endothelial phenotypes of blood and lymphatic capillaries. Am J Pathol 154: 385-394, 1999.

23. Fukunaga M: Expression of D2-40 in lymphatic endothelium of normal tissues and in vascular tumours. Histopathology 46: 396-402, 2005.

24. Kahn HJ, Bailey D and Marks A: Monoclonal antibody D2-40, a new marker of lymphatic endothelium, reacts with Kaposi's sarcoma and a subset of angiosarcomas. Mod Pathol 15: 434-440, 2002 .
25. Naqvi J, Ordonez NG, Luna MA, Williams MD, Weber RS and El-Naggar AK: Epitheloid hemangioendothelioma of the head and neck: role of podoplanin in the differential diagnosis. Head Neck Pathol 2: 25-30, 2007

26. Fujii T, Zen Y, Sato Y, et al: Podoplanin is a useful diagnostic marker for epithelioid hemangioendothelioma of the liver. Mod Pathol 21: 125-130, 2008.

27. Roy S, Chu A, Trojanowski JQ and Zhang PJ: D2-40, a novel monoclonal antibody against the M2A antigen as a marker to distinguish hemangioblastoma from renal cell caricinomas. Acta Neuropathol 109: 497-502, 2005.

28. Sonne SB, Herlihy AS, Hoei-Hansen CE, et al: Identity of M2A (D2-40) antigen and gp36 (Aggrus, T1A-2, podoplanin) in human developing testis, testicular carcinoma in situ and germ-cell tumours. Virchows Arch 449: 200-206, 2006.

29. Zayed AE, Abd-Elnaeim MM, Abd-Elghaffar SKh, Hild A, Brehm R and Steger K: Prenatal development of murine gonads with special reference to germ cell differentiation: a morphological and immunohistochemical study. Andrologia 39: 93-100, 2007.

30. Mishima K, Kato Y, Kaneko MK, Nishikawa R, Hirose T and Matsutani M: Increased expression of podoplanin in malignant astrocytic tumors as a novel molecular marker of malignant progression. Acta Neuropathol 111: 483-488, 2006.

31. Mishima K, Kato Y, Kaneko MK, et al: Podoplanin expression in primary central nervous system germ cell tumors: a useful histological marker for the diagnosis of germinoma. Acta Neuropathol 111: 563-568, 2006.

32. Shibahara J, Kashima T, Kikuchi Y, Kunita A and Fukayama M: Podoplanin is expressed in subsets of tumors of the central nervous system. Virchows Arch 448: 493-499, 2006.

33. Durchdewald M, Guinea-Viniegra J, Haag D, et al: Podoplanin is a novel fos target gene in skin carcinogenesis. Cancer Res 68: 6877-6883, 2008

34. Martín-Villar E, Scholl FG, Gamallo C, Yurrita MM, MuñozGuerra M, Cruces J and Quintanilla M: Characterization of human PA2.26 antigen (T1alpha-2, podoplanin), a small membrane mucin induced in oral squamous cell carcinomas. Int J Cancer 113: 899-910, 2005.

35. Yuan P, Temam S, El-Naggar A, Zhou X, Liu DD, Lee JJ and Mao L: Overexpression of podoplanin in oral cancer and its association with poor clinical outcome. Cancer 107: 563-569, 2006.

36. Kawaguchi H, El-Naggar AK, Papadimitrakopoulou V, et al: Podoplanin: a novel marker for oral cancer risk in patients with oral premalignancy. J Clin Oncol 26: 354-360, 2008.

37. Liang $\mathrm{H}, \mathrm{Wu} \mathrm{H}$, Giorgadze TA, et al: Podoplanin is a highly sensitive and specific marker to distinguish primary skin adnexal carcinomas from adenocarcinomas metastatic to skin. Am J Surg Pathol 31: 304-310, 2007.

38. Yu H, Pinkus GS and Hornick JL: Diffuse membranous immunoreactivity for podoplanin ( D2-40) distinguishes primary and metastatic seminomas from other germ cell tumors and metastatic neoplasms. Am J Clin Pathol 128: 767-775, 2007.

39. Jokinen CH, Dadras SS, Goldblum JR, van de Rijn M, West RB and Rubin BP: Diagnostic implications of podoplanin expression in peripheral nerve sheath neoplasms. Am J Clin Pathol 129: 886-893, 2008

40. Martín-Villar E, Megías D, Castel S, Yurrita MM, Vilaró S and Quintanilla M: Podoplanin binds ERM proteins to activate RhoA and promote epithelial-mesenchymal transition. J Cell Sci 119: 4541-4553, 2006.

41. Scholl FG, Gamallo C, Vilar S and Quintanilla M: Identification of PA2.26 antigen as a novel cell-surface mucin-type glycoprotein that induces plasma membrane extensions and increased motility in keratinocytes. J Cell Sci 112: 4601-4613, 1999.

42. Yu Y, Khan J, Khanna C, Helman L, Meltzer PS and Merlino G: Expression profiling identifies the cytoskeletal organizer ezrin and the developmental homeoprotein Six-1 as key metastatic regulators. Nat Med 10: 175-181, 2004.

43. Ariizumi T, Ogose A, Kawashima H, et al: Expression of podoplanin in human bone and bone tumors: New marker of osteogenic and chondrogenic bone tumors. Pathol Int 60: 193-202, 2010.

44. Suzuki H, Kato Y, Kaneko MK, Okita Y, Narimatsu H and Kato M: Induction of podoplanin by transforming growth factor- $\beta$ in human fibrosarcoma. FEBS Lett 582: 341-345, 2008 